\title{
Evaluation of water structures in cotton cloth by fractal analysis with broadband dielectric spectroscopy
}

\author{
Shin Yagihara ${ }^{1, *}$ (D), Hironobu Saito ${ }^{1}$, Hironori Sugimoto ${ }^{1}$, Tsubasa Kawaguchi ${ }^{1}$, \\ Minoru Fukuzaki ${ }^{2}$, Takako Igarashi ${ }^{3}$, Masato Hoshi ${ }^{4}$, and Koichi Nakamura ${ }^{3}$ \\ ${ }^{1}$ School of Science, Tokai University, Hiratsuka-shi, Kanagawa 259-1292, Japan \\ ${ }^{2}$ Liberal Arts Education Center, Tokai University, Kumamoto-shi, Kumamoto 862-8652, Japan \\ ${ }^{3}$ Material Science Research Laboratory, Kao Corporation, Wakayama-shi, Wakayama 640-8580, Japan \\ ${ }^{4}$ Analytical Science Research Laboratory, Kao Corporation, Wakayama-shi, Wakayama 640-8580, Japan
}

Received: 9 June 2021

Accepted: 4 August 2021

Published online:

27 August 2021

(C) The Author(s) 2021

\begin{abstract}
Broadband dielectric spectroscopy measurements were performed on naturally dried cotton cloth, and a recently developed analytical technique for fractal analysis of water structures was applied to obtain existential states and locations of water molecules in the material. Three relaxation processes observed in $\mathrm{GHz}$, $\mathrm{MHz}$, and $\mathrm{kHz}$ frequency regions were attributed to dynamic behaviors of hydrogen bonding networks (HBNs) of water and interacting molecules, polymer chains with interacting ion and water molecules, and ions restricted on the interfaces of larger structures, respectively. Water molecules were heterogeneously distributed in the cotton cloth, and the HBNs remained as a broad $\mathrm{GHz}$ frequency process. Fractal analysis suggested that water molecules distributed in the material were characterized by a small value (0.55) of the Cole-Cole relaxation time distribution parameter, indicating spatial distribution of HBN fragments with various sizes in cotton cloth. This result was also supported by the $T_{2}$ relaxation time obtained from nuclear magnetic resonance for naturally dried cotton yarn. Comparing previous results of dielectric relaxation measurements and fractal analysis with the $\tau-\beta$ diagram for various aqueous systems, the results determined that water molecules cannot exist inside cellulose microfibrils. The fractal analysis employed in this work can be applied to dynamic water structures in any material. The presented analytical technique with a universal $\tau-\beta$ diagram is expected to be an effective tool to clarify water structure detail even for heterogeneous hydrations of the low water content substances.
\end{abstract}

Handling Editor: Stephen Eichhorn.

Address correspondence to E-mail: yagihara@keyaki.cc.u-tokai.ac.jp 


\section{GRAPHICAL ABSTRACT}

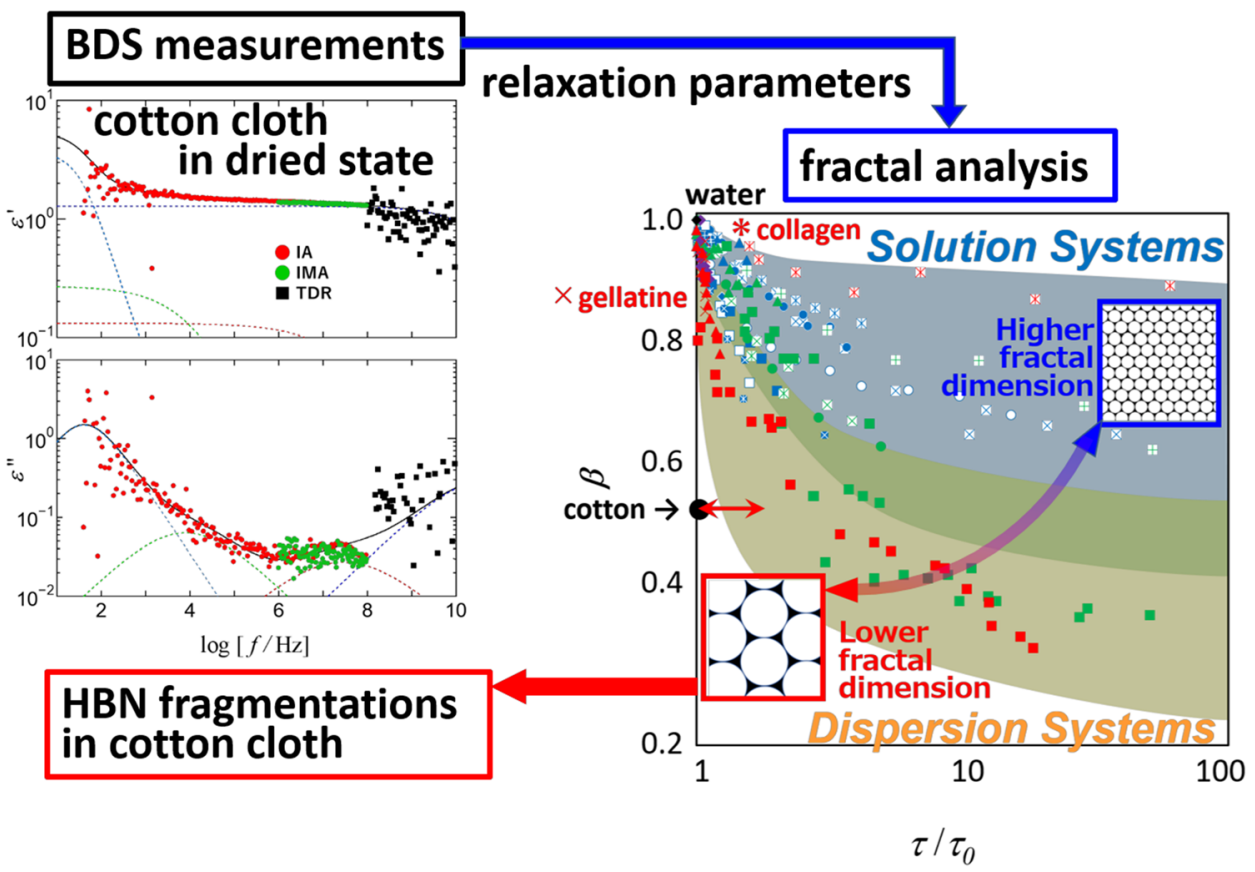

\section{Introduction}

Cotton materials are naturally derived substances used in our daily lives. Since the structure and physical property are affected by the water content, the hydration structure and mechanism in cotton [1-3] and the main component, cellulose [4-9] and related materials [10, 11] have been extensively studied. However, since water molecules form various water structures interacting with surrounding molecules form various water structures, there exist difficulties in observation and analyzing those behaviors in detail, and various approaches are still required.

One of the authors (T. I.) has recently presented a hydration model of cotton cloth to explain the hardening effect of dry cotton rag from the wet state [1-3]. The hardening effect disappears with mechanical stimulus, and it is not shown in the completely dry state. These results suggest that the hardening effect brought by water molecules is not explained by only the average water content but also the spatial distribution of water molecules in the dry sample. The model shows that the stiffness of cotton cloth is caused by a cross-linked network between single fibers mediated by capillary adhesion of bound water on the surface of cellulose. However, it is difficult to evaluate the spatial distribution of water molecules in a low water content sample especially for such heterogeneous materials.

Broadband dielectric spectroscopy (BDS) [12] is recognized as one of most effective tools to study hydration structures of various moist materials, including biological systems [13, 14]. The $\mathrm{GHz}$ relaxation process, generally observed for water [15-17] and aqueous polymer systems [18-21], exhibits a decrease in the relaxation frequency with decreasing water content. In other words, water molecules involved in moist materials, such as polymer-water systems, show a plasticizer effect in which water molecules increase mobility of both water molecules and local polymer chains with increase in water content [21, 22]. Decreases in molecular mobility, typically observed with reduced water content and temperature, are often accompanied by 
structural formations called slow dynamics. Dielectric measurements for the $\mathrm{GHz}$ process of aqueous materials provide meaningful information about dynamic behaviors of water structures. Dielectric spectroscopy has been applied to hydration studies on cellulose [9] and related materials [10, 11]. Zhao et al. reported that BDS and nuclear magnetic resonance (NMR) measurements of wide regions of temperature and water content suggested various states of restricted water molecules [9].

In the low water content region, however, relaxation processes with low dielectric constant become broader, and their overlap tends to induce ambiguities and make systematic and universal interpretation difficult especially for heterogeneous materials like cotton cloth. The problem is even more common for low water content substances. Therefore, it is desired to apply an effective approach that can be utilized to clarify various water structures for any materials in combination. There are few universal and accurate methodologies for comparing the hydration processes of materials with different chemical structures other than fractal analysis applied to $\mathrm{GHz}$ frequency process observed by dielectric spectroscopy measurements for moist substances [23-27]. The relaxation mechanism of the $\mathrm{GHz}$ frequency process has been explained by cooperative exchanges in hydrogen bonding networks (HBNs) for hydrogen bonding liquid and mixtures [21, 28]. Fractal behaviors characterized by the lower fractal dimension were usually shown for more heterogeneous systems [14, 29, 30]. This result means that HBNs tend to remain even in the lower water content region for more heterogeneous systems and the analysis suggests an application to evaluate the spatial distribution of water molecules in HBN fragments. Details of the physical meaning and application of fractal analysis are described in the following section.

In the present study, we examined a practical methodology of fractal analysis with the BDS measuring technique to clarify spatial distributions and fluctuations of water molecules in HBN fragments for cotton cloth. The water structures thus obtained by fractal analysis were also confirmed by the transverse relaxation time, $T_{2}$, obtained by NMR measurements for cotton yarn. The consistency of the hydration model is also considered. Combinations of these evaluation techniques at different scales of observation are also expected to offer additional information for obtaining detailed water structures [14, 29-31].

\section{Fractal analysis of water structures}

\section{Conventional fractal analysis of water structures with dielectric spectroscopy}

Mixtures of polymers and hydrogen bonding solvents, like water, exhibit a $\mathrm{GHz}$ frequency process and another process, observed at frequencies around 100 times lower at typical temperatures, due to chain dynamics [21]. The $\mathrm{GHz}$ frequency process is often described by symmetric relaxation curves described by the Cole-Cole relaxation function [32],

$\frac{\varepsilon^{*}-\varepsilon_{\infty}^{\prime}}{\varepsilon_{S}^{\prime}-\varepsilon_{\infty}^{\prime}}=\frac{1}{1+(j \omega \tau)^{\beta}}, \quad(0<\beta \leq 1)$,

where the complex dielectric constant, $\varepsilon *\left(=\varepsilon^{\prime}-j \varepsilon\right)$, is normalized by the relaxation strength, $\Delta \varepsilon\left(=\varepsilon_{s}^{\prime}-\varepsilon_{\infty}^{\prime}\right) ; \varepsilon_{s}^{\prime}$ and $\varepsilon_{\infty}^{\prime}$ are the low and high-frequency limits of the dielectric constant, respectively; $j$ is the imaginary unit; $\omega$ is the angular frequency; $\tau$ is the relaxation time; and $\beta$ is the Cole-Cole symmetric relaxation time distribution parameter. When the $\beta$ value is unity, the relaxation process is described by the Debye equation [33]. By contrast, the lower-frequency process due to local chain dynamics of polymers often exhibits asymmetric curves described using the Kohlrausch-Williams-Watts function [34], Havriliak-Negami function [35], etc.

However, both processes are often affected by opposite components because of the inter-molecular interactions. Thus, the high-frequency process also reflects the local parts of the chain, and the low-frequency process also contains solvent molecules attached to the polymer chains. Since the degree of these interactions is dependent on the respective mixtures, there still exist a lot of investigations on liquid structures. The $\mathrm{GHz}$ frequency process can sometimes be treated by two or three Debye-type processes for precise fitting procedures.

In the present work, we characterize fluctuation of dynamic behaviors of HBNs and dispersion of their locations as a hydration model of cotton cloth. In this case, it is not suitable to allocate many Debye processes to express a broad relaxation process, even for materials with low water content. The explanation of liquid structures by several Debye processes with similar relaxation times is not suitable for the heterogeneous and fluctuating structures of liquids treated in the present work. 
Generally, conventional analysis of water structures using dielectric spectroscopy for aqueous polymer solutions examines three relaxation parameters $(\tau, \beta$, and $\Delta \varepsilon)$ for the $\mathrm{GHz}$ relaxation process as independent variables. Figure $1 \mathrm{a}$ and $\mathrm{b}$ shows typical model behaviors of water content dependence for relaxation time, $\tau$, and its distribution parameter, $\beta$ respectively. However, the common solvent, water, in the mixtures should exhibit characteristic trajectories of slow dynamics with decrease in water content, as shown in Fig. 1c [14, 19]. Therefore, water content dependencies of the relaxation parameters, shown in Fig. 1a and b, cannot be sufficiently characterized for water structures. The relationship between the parameters should be determined to characterize the water structures shown in Fig. 1c and their physical meaning.

The relationship between the parameters can be described from a simple ergodic hypothesis for the time response function reflecting geometric fractal structures [23, 24],

$\beta=\frac{d_{G}}{2} \frac{\ln \left(\tau \omega_{S}\right)}{\ln \left(\tau / \tau_{0}\right)}$,

where $\tau_{0}$ is the cutoff time of the scaling in the time domain and $d_{\mathrm{G}}$ is the fractal dimension of the point set where relaxing units are interacting with the statistical reservoir. In Eq. (2),

$\omega_{S}=2 d_{E} G^{2 / d_{G}} D_{S} / R_{0}^{2}$
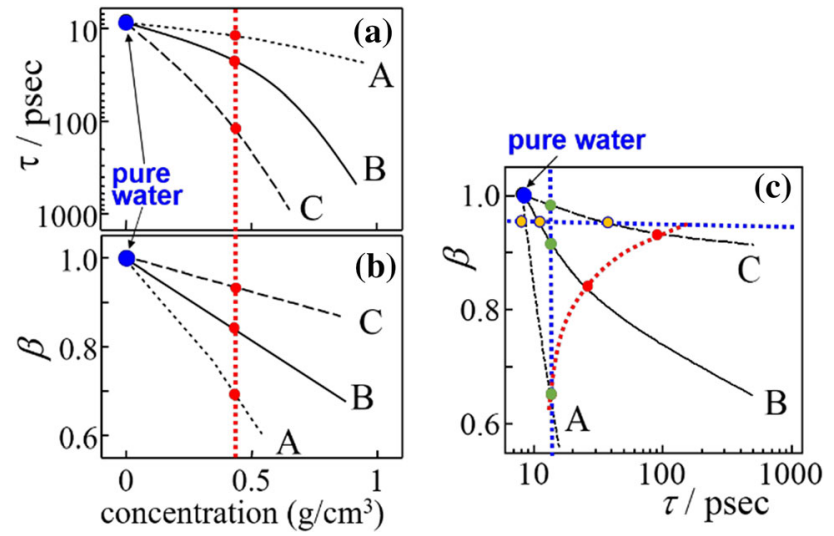

Figure 1 Examples of concentration dependences of a relaxation time and $\mathbf{b}$ the relaxation time distribution parameter for model materials, A, B, and C. c The relaxation time dependence of its distribution parameter for the same model materials. Red and blue dotted lines represent conventional and current analysis, respectively. (Adapted from Fig. 3 in [30]). is the characteristic frequency of the self-diffusion process, where $d_{\mathrm{E}}$ is the Euclidean dimension, $D_{\mathrm{s}}$ is the self-diffusion coefficient, $R_{0}$ is the cut-off size of the scaling in the space, and $G$ is a geometrical coefficient approximately equal to unity.

Equation (2) indicates a hyperbolic relation between the average relaxation time and its distribution parameter, as shown in Fig. 1c. The $\tau-\beta$ diagram of the fractal analysis suggests a reasonable comparison of the relaxation time distribution parameter at the same average relaxation time for any aqueous system. The intercept on the vertical axis of the asymptote parallel to the horizontal axis of the hyperbola in the figure corresponds to the fractal dimension. Curve $C$ shows a higher value of fractal dimension than do curves A and B. It is clearly indicated that the result of fractal analysis in Fig. 1c may be quite different from the conventional water content dependencies discussed using Fig. $1 a$ and $b$.

Although the physical meanings obtained from fractal analysis are important, these treatments are not easy since accurate dielectric measurements in a wide frequency range, from higher to lower frequencies, are difficult, especially in the case of materials with low water content. In addition, the fractal dimension obtained by the analysis often appears to be a nonsensical result, such as the small value (less than unity) usually shown for protein solutions. However, recent applications of fractal analysis for various moist materials, including biological materials and cement materials, have solved this problem and made fractal analysis a practical analytical technique for water structures [14, 30, 36].

\section{Recent developments of fractal analysis}

A physical picture of $\mathrm{HBN}$ dynamics for the molecular mechanism of the $\mathrm{GHz}$ frequency process was supported by dielectric studies of hydrogen bonding materials [21, 28]. The concept of HBNs also clarifies how a water structure should be considered by fractal analysis [30]. When fractal analysis was first considered, two-dimensional networks of hydrogen bonds among water molecules were discussed, and Eqs. 2 and 3 were derived since the maximum value of the fractal dimension was $2[23,24]$. Though this value can be easily modified by the correction parameter, we maintain the original form here. In fact, various fractal analyses extensively developed 
since the 1980s have generally been considered less suitable for characterization after detailed evaluation of the physical properties of substances. Then, in actual analyses, the relative values and their changes are usually much more important than the absolute value of the fractal dimension.

The HBN dynamics model suggests that the molecular mechanism of the $\mathrm{GHz}$ process is a rate process of exchanges in $\mathrm{HB}$ and the relaxation time becomes larger with decrease in number density of HBs cite. Then, the physical pictures of restricted dynamics of bound water molecules, often shown for hydration studies, suggest that the water molecules are not restricted by the HB itself but by the decrease in the number density of $\mathrm{HB}$ sites [21, 28]. This property also corresponds to the plasticizer effect of water molecules to interacting polymer chains [21, 22].

It has been recently confirmed that the value of the fractal dimension of HBNs could be determined from the box-counting method [14]. In general, the value of fractal dimension determined by observations is dependent on the length scale used in the observation technique. Using observation techniques with small length scales, any structure should have a maximum fractal dimension of 3 . In that sense, there exist no absolute values of fractal dimension, and characterization of structures is always obtained as relative values of fractal dimension dependent on the observation techniques. This universal property of fractal analysis is also available, even in cases that do not deal directly with length scales, such as dielectric spectroscopy [30]. Since length and time scales are indirectly interrelated through the dynamic nature of complex systems, no observation method can be completely independent from both length and time scales. Therefore, fractal analysis by dielectric spectroscopy suggests that the trajectories shown in Fig. 1c reflect how the HBN is fragmented and how water molecules are dispersed in materials.

As a result of the above confirmation of the characteristic feature of fractal analysis, an advanced analytical technique can be conducted by using the results we have obtained thus far. Even if the absolute value of the fractal dimension is not determined from analysis of the hyperbola in Fig. 1c, the possible value of the fractal dimension can be estimated from the location of plots and a single plot on the $\tau-\beta$ diagram. The existence state of water molecules can then be discussed as the degree of HBN fragmentations. Using previous fractal analysis obtained for various aqueous systems, even a single plot on the $\tau-\beta$ diagram can characterize the water structure from comparisons with various trajectories. The methodology of fractal analysis is also available for other symmetric relaxation processes with relaxation mechanisms observed in lower frequencies, such as structured water molecules [36] and ion dynamics on the interface [37]. These examinations make fractal analysis more universal and an effective analytical technique.

\section{Materials and methods}

\section{Preparation of cotton cloth and yarn}

Plain-woven parts of cotton towels (TW220, Takei Corp. Japan) were used as sample cloth. Before the experiment, these fibers were pre-washed completely using two methods (referred to as methods A and B) to remove all fiber-treatment agents used to manufacture the fibers. After pre-washing, the cotton cloths were purified with a mixed bed type of ionexchanged resins (501-X8, manufactured by Bio-Rad, USA) to remove ionic components, such as $\mathrm{Ca}^{2+}$, $\mathrm{Mg}^{2+}, \mathrm{Na}^{+}$, and $\mathrm{K}^{+}$, in the cloth. Elemental analysis after purification was below $10 \mathrm{mg} \mathrm{kg}^{-1}$.

In Method A, samples were pre-washed using a fully automatic washing machine (NA-F702P, Panasonic Corp. Japan). Twenty-four cotton towels and $52.22 \mathrm{~g}$ of nonionic detergent (Emulgen108, Kao Corp. Japan, $10 \%$ aqueous solution) were put into the washing machine with $47 \mathrm{~L}$ of water and washed according to the following two steps: (1) samples were washed for $9 \mathrm{~min}$ (with water containing the above-mentioned nonionic detergent), rinsed twice with water, and spin-dried for $3 \mathrm{~min}$ (this step was repeated thrice); and (2) samples were then washed for 9 min (with water only), rinsed twice with water, and spun dried for $3 \mathrm{~min}$ (this step was repeated twice).

In Method B, samples were pre-washed with organic solvents. Cotton towels were first cut into pieces $(8 \mathrm{~cm} \times 8 \mathrm{~cm})$ and pre-washed using Method A. Then, the samples were washed again and stirred in $300 \mathrm{~mL} \mathrm{CHCl}_{3} / \mathrm{MeOH}$ (1:1 wt. ratio) for $5 \mathrm{~min}$ in a beaker; this process was repeated 5 times. For polyester faille and cotton yarns, only solvent washing was applied. 
For a desalination procedure, the same weight of ion exchange resin (AG®501-X8, Bio-Rad) as the cotton samples was added to $2 \mathrm{~L}$ of ultrapure water (Milli-Q water, Millipore) and stirred for 1 or 10 days. The obtained were then air-dried on a clean bench with $45 \%$ relative humidity ( $\mathrm{RH}$ ) for about 5 days.

Samples were kept in the laboratory at a constant temperature and humidity of $23 \pm 1{ }^{\circ} \mathrm{C}$ and $50 \% \pm 1 \% \mathrm{RH}$ before all measurements.

\section{Broadband dielectric relaxation measurements for cotton cloth}

High-frequency dielectric spectroscopy measurements were performed by our original time domain reflectometry (TDR) method with a digitizing oscilloscope mainframe (86100C, Agilent Technologies) for wet samples in the frequency range of $100 \mathrm{MHz}$ up to $30 \mathrm{GHz}$. For dried samples, another mainframe (54120B, Hewlett-Packard) with Four Channel Test Set (HP 54124A) in the frequency range of $100 \mathrm{MHz}$ up to $10 \mathrm{GHz}$ was used. The electrode used for the present work was handmade open-ended coaxial electrodes with an outer conductor having an outer diameter of $2.2 \mathrm{~mm} \varphi$. After calibration procedures, measurements were performed with ultrapure water (Milli-Q, Millipore) and 1, 4-dioxane for wet and dried reference samples, respectively. The flat end of a semirigid coaxial electrode was set up to have good contact with the sample surface, and dielectric measurements were performed three times for each sample to confirm the reproducibility of the contact at $21 \pm 1{ }^{\circ} \mathrm{C}$ and $50 \pm 1 \%$ RH. Details on dielectric relaxation measurements with TDR systems were described in our previous papers [14, 30, 38].

For dielectric measurements in the lower and middle frequency regions, an Alpha-A-Analyzer (Novocontrol, $10 \mathrm{mHz}$ to $10 \mathrm{MHz}$ ), LCR meter (E4980E, Agilent Technologies, $20 \mathrm{~Hz}$ to $3 \mathrm{MHz}$ ), impedance analyzer (IA: 4294A, Agilent Technologies, $40 \mathrm{~Hz}$ to $110 \mathrm{MHz}$ ), and impedance/material analyzer (IMA: HP4291B, Hewlett-Packard, $1 \mathrm{MHz}$ to $1.8 \mathrm{GHz}$ ) were employed.

To examine the effect of deionization, an Alpha-AAnalyzer with handmade three-terminal parallelplate electrodes (diameter: $10 \mathrm{~mm} \varphi$ ) and an LCR meter with three-terminal parallel-plate electrodes (A-type electrode with a diameter of $38 \mathrm{~mm} \varphi$, 16451B, Hewlett-Packard) were used. Cotton cloth samples set into the electrodes were placed in a chamber regulated to constant humidity/temperature, as shown in Fig. 2. The $C_{0}$ values were approximately 1.4 and $20 \mathrm{pF}$ for the Alpha-A-Analyzer and LCR meter, respectively.

For other measurements, IA and IMA measurements were performed with handmade two-terminal parallel-plate electrodes inside the SMA-type connector, as shown in Fig. 3. The $C_{0}$ value was about $0.45 \mathrm{pF}$.

\section{$T_{2}$ relaxation measurements for cotton cloth relaxation and measurements of nuclear magnetic resonance for cotton yarns}

NMR measurements were performed by a $400-\mathrm{MHz}$ NMR (Ascend 400WB, Bruker) with a 5-mm-diameter NMR tube to obtain the $T_{2}$ relaxation time for cotton yarn samples. The Carr-Purcell-Meiboom-Gill (CPMG) method [39] was used to determine the $T_{2}$ relaxation time with 32 times integration. After acquisition of the background signals, the difference from the obtained sample signal was analyzed.

The cotton yarns were kept in a constant temperature and humidity chamber at $50 \pm 5 \% \mathrm{RH}$ and $23 \pm 2{ }^{\circ} \mathrm{C}$ for about 1 week in advance. For preparation of humidified samples, one end of the sample

(a)

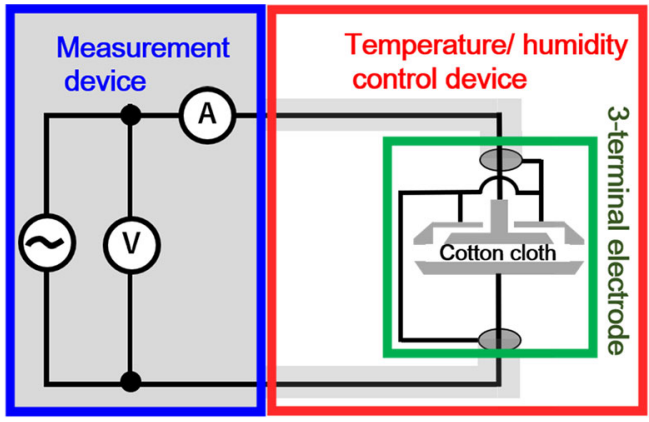

(b)

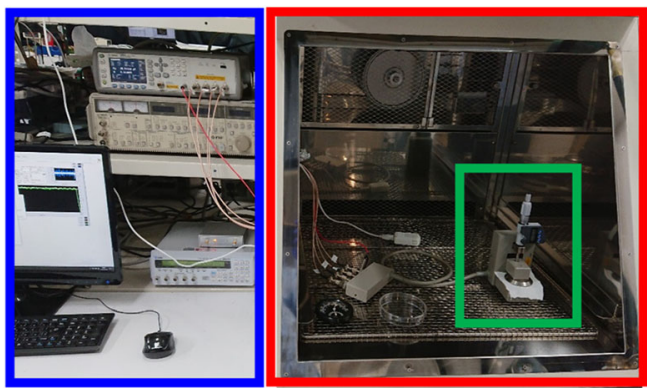

Figure 2 BDS measurement system with measurement devise (blue frame) for cotton cloth samples controlled at constant temperature and humidity in chamber (red frame) with threeterminal parallel-plate electrodes (green frame): a schematic diagram and $\mathbf{b}$ photograph of the entire system. 


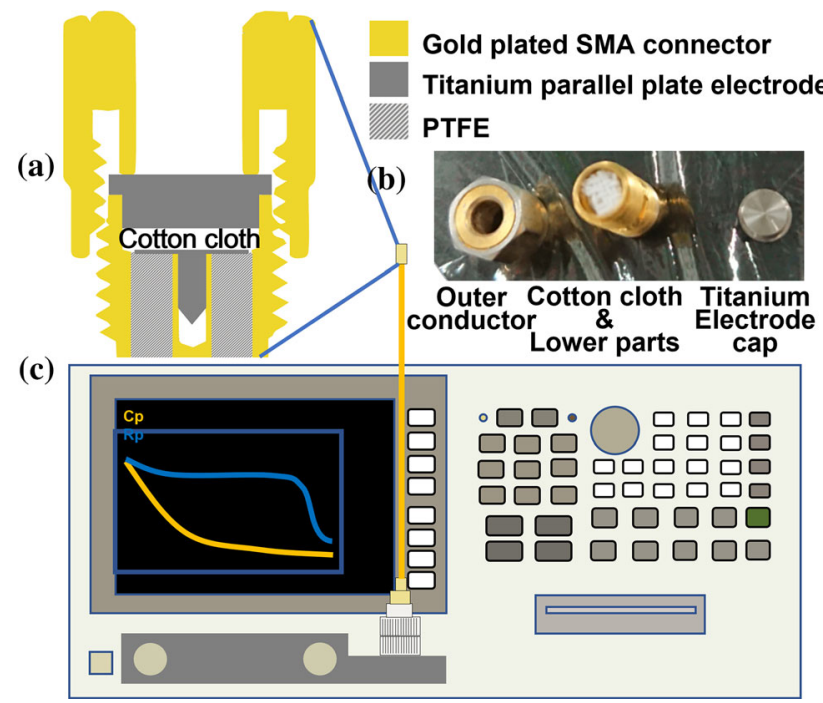

Figure 3 Dielectric relaxation measuring system for cotton cloth samples with handmade two-terminal parallel-plate electrodes inside the SMA connector: a cross section, $\mathbf{b}$ photograph of parts, and $\mathrm{c}$ mainframe.

tube was cut and both ends were opened; the sample tube was kept in a desiccator with $98 \% \mathrm{RH}$ for 1 week. The tube was sealed again with silicon putty before NMR measurements. Averaged results were obtained by using three to eight samples under each condition. In addition, some samples were vacuumdried at room temperature for about 1 week and then allowed to stand in a constant temperature and humidity chamber again to confirm reproducibility.

\section{Results and discussion}

Figure 4 shows relaxation curves obtained by TDR measurements for wet cotton cloths with different numbers of sheets. The sheet samples were placed on an open-ended electrode facing up. In the case of a small number of sample sheets, smaller permittivity was observed as the electric field pattern passed through the sample and reached the air. This result suggested that at least five sheets are necessary for TDR measurements. Then, eight sheets obtained by folding three times were used in the following measurements.

As shown in Fig. 4, a $\mathrm{GHz}$ frequency process at around $10 \mathrm{GHz}$ and a higher frequency tail of the $\mathrm{MHz}$ frequency process were observed. Considering the typical experimental results obtained for moist materials in the frequency range, the $\mathrm{GHz}$ frequency

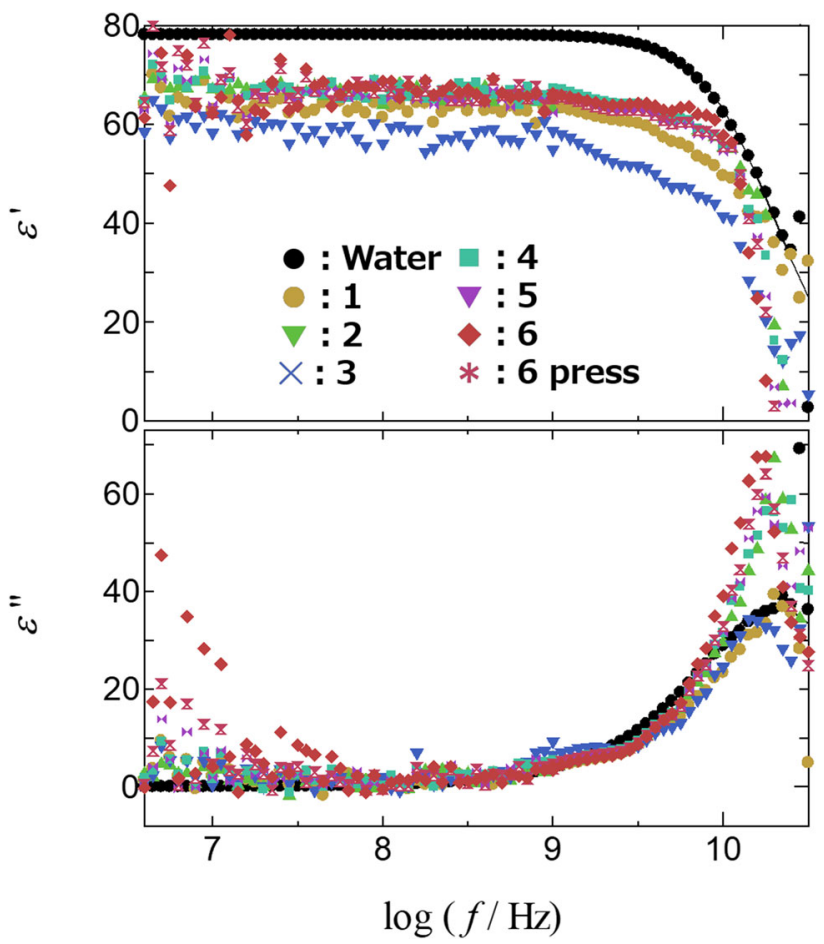

Figure $4 \mathrm{GHz}$ frequency process with the higher frequency tail of the $\mathrm{MHz}$ frequency process observed by TDR measurements for cloth samples. The numbers in the figure indicate the number of cloth sample sheets.

process is attributed to cooperative dynamics of water molecules. In the present paper, the molecular mechanism of HBN dynamics is considered for the $\mathrm{GHz}$ frequency process.

Figure 5 shows relaxation curves obtained by lower-frequency measurements for naturally dried samples of cotton cloths with and without deionization procedure. The relaxation curves clearly show that ion dynamics have enlarged contributions of dc conductivity and all relaxation processes including the higher frequency processes, and respective processes must be obscured by the ionic process without deionization. Thus, samples of cotton cloths used in the present study were sufficiently deionized before measurements.

Figures 4 and 5 show three relaxation processes observed in the $\mathrm{GHz}, \mathrm{MHz}$, and $\mathrm{kHz}$ frequency regions and another process due to electrode polarization in the lowest frequency region, even for dry cotton cloth samples. These results are supported by a recent work by Zhao et al. [9], even though they analyzed more detailed fitting analysis for each process in wide temperature and water content regions. Part of their results for cellulose samples seems to 


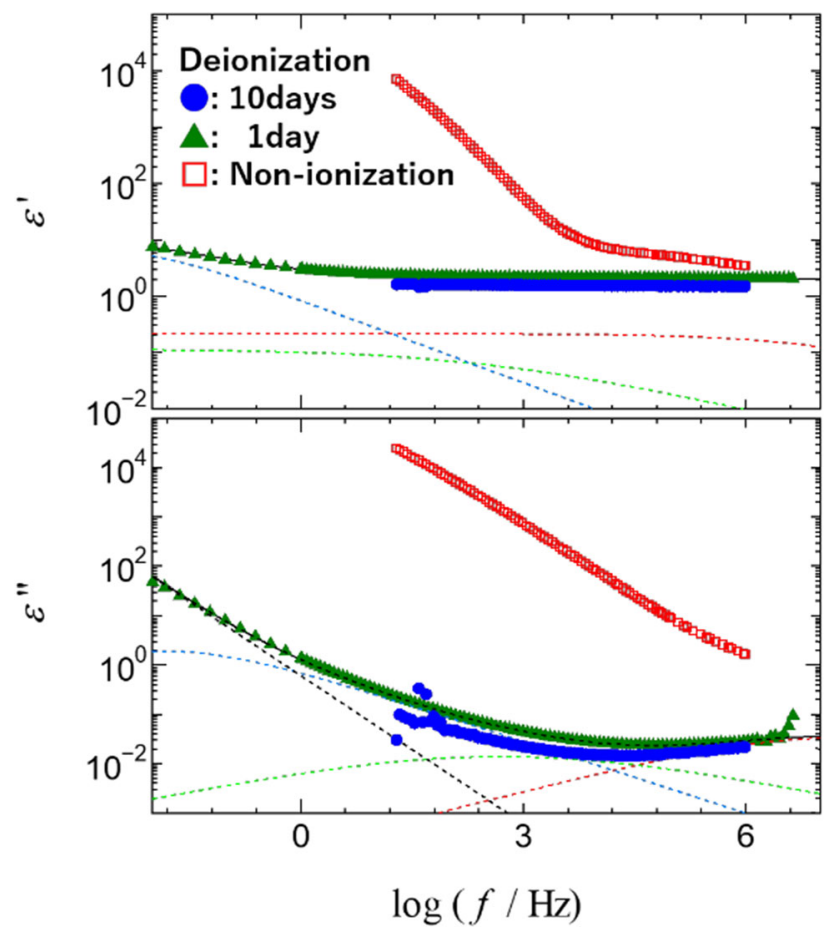

Figure 5 Lower-frequency processes observed for dried samples of cotton cloths with and without deionization. The dotted lines show respective processes due to dc conductivity, electrode polarization, and interfacial polarization from the lower-frequency side.

reflect some ion dynamics. Our recent work also indicated that these ionic behaviors on interfaces can be observed, even in the $\mathrm{MHz}$ frequency region, for small-scale heterogeneities [37].

Measurement error is inevitable, especially around $1 \mathrm{MHz}$ in BDS measurements since the measurement principals of higher frequency transmission line and lower-frequency electric circuit measurements are essentially different and switch around $1 \mathrm{MHz}$. In addition, hard materials with low dielectric loss and wide relaxation time distributions in the dry state make measurement and analysis difficult because of contact problems between electrodes and samples with large-scale heterogeneities of cotton cloth.

Therefore, in Fig. 6, the value of the dielectric constant, $\varepsilon_{10}^{\prime} \mathrm{MHz}$, on the highest frequency side $(\sim 10 \mathrm{MHz})$ obtained in Fig. 5 was plotted against the water content. The high-frequency limit of the dielectric constant in the lower-frequency measurements describes the remaining relaxation strength for high-frequency processes in the higher frequency region. Compared with the proportional relationship, indicated by the dashed line for high water content

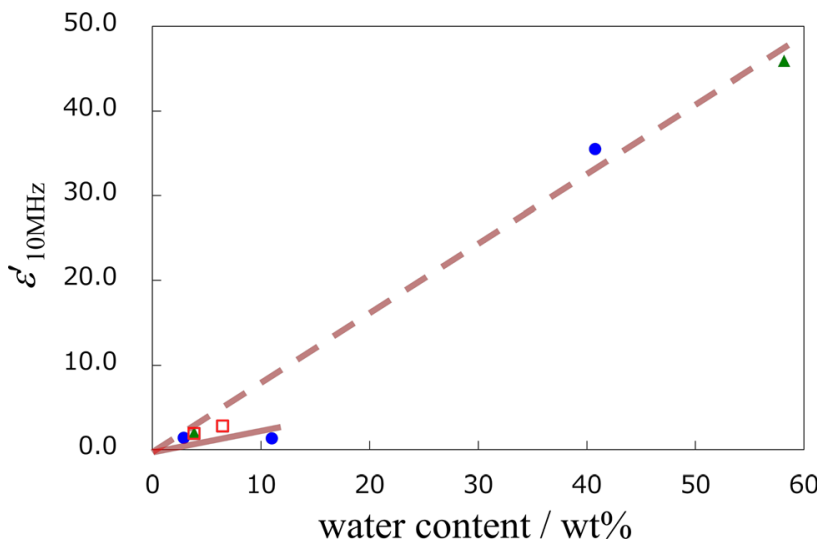

Figure 6 Water content dependency of the dielectric constant, $\varepsilon^{\prime \prime}{ }_{10 \mathrm{MHz}}$, at the higher frequency side $(\sim 10 \mathrm{MHz})$ for the lower relaxation processes. Dashed and solid lines were obtained for humid and dried samples, respectively.

samples, the solid line obtained for plots of the low water content samples clearly shows a smaller slope. Even while considering the presence of air in the low water content sample, the dielectric constant still seems small. This result indicates that there is another relaxation process on the high-frequency side, in addition to the relaxation process observed in the frequency domain up to the $\mathrm{MHz}$ region. As is often seen in dispersion systems, the relaxation process due to dynamic behaviors of HBNs of water with cellulose molecules observed in the $\mathrm{GHz}$ frequency region for the cotton cloth sample widens the tail on the lower-frequency side, maintaining the average relaxation time.

Recent understanding in the relaxation mechanism of the $\mathrm{GHz}$ frequency process of water with $\mathrm{HBNs}$ can be associated with a model wherein water molecules heterogeneously distributed in the cotton cloth exhibit a broad relaxation process. However, the dielectric properties of dry cotton cloths have not yet been clarified in detail, especially between the $10 \mathrm{GHz}$ range and $10 \mathrm{MHz}$ range processes. This is because of the difficulty that observation techniques have in replacing measuring principals and ambiguities in heterogeneous hydration. It is difficult to expect an effective improvement in measurement accuracy at present, and it is difficult to capture some changes in the hydration state with clear relaxation peaks, especially for low water content samples. Therefore, it is effective to confirm how $\mathrm{GHz}$ frequency data continue to the lower-frequency region 
and to perform data analysis that makes full use of the characteristics of broadband data.

Another series of dielectric measurements, in a middle frequency region from $1 \mathrm{MHz}$ up to $1 \mathrm{GHz}$, were added to compensate a part of the spectra in Fig. 7, which shows a BDS spectrum for a dry sample of cotton cloth. Plots for three independent observations are shown for a $\mathrm{GHz}$ frequency process obtained from TDR measurements. Though data scattering and systematic errors appearing between the two different series of measuring systems were eliminated at $\sim 1 \mathrm{MHz}$, experimental errors at $\sim$ $100 \mathrm{MHz}$ with large scattering from TDR measurements could not be sufficiently reduced. Since a lowloss sample of 1,4-dioxane is used as the standard sample for calibration in dielectric measurements, the complex dielectric constant thus obtained indicated the existence of the $\mathrm{GHz}$ frequency process, although large scattering of data was observed. The error in the measurement data is mainly due to the low quality of the contact between the small size electrodes required for high-frequency measurements and the cotton cloth sample. This is unavoidable, especially in a fiber

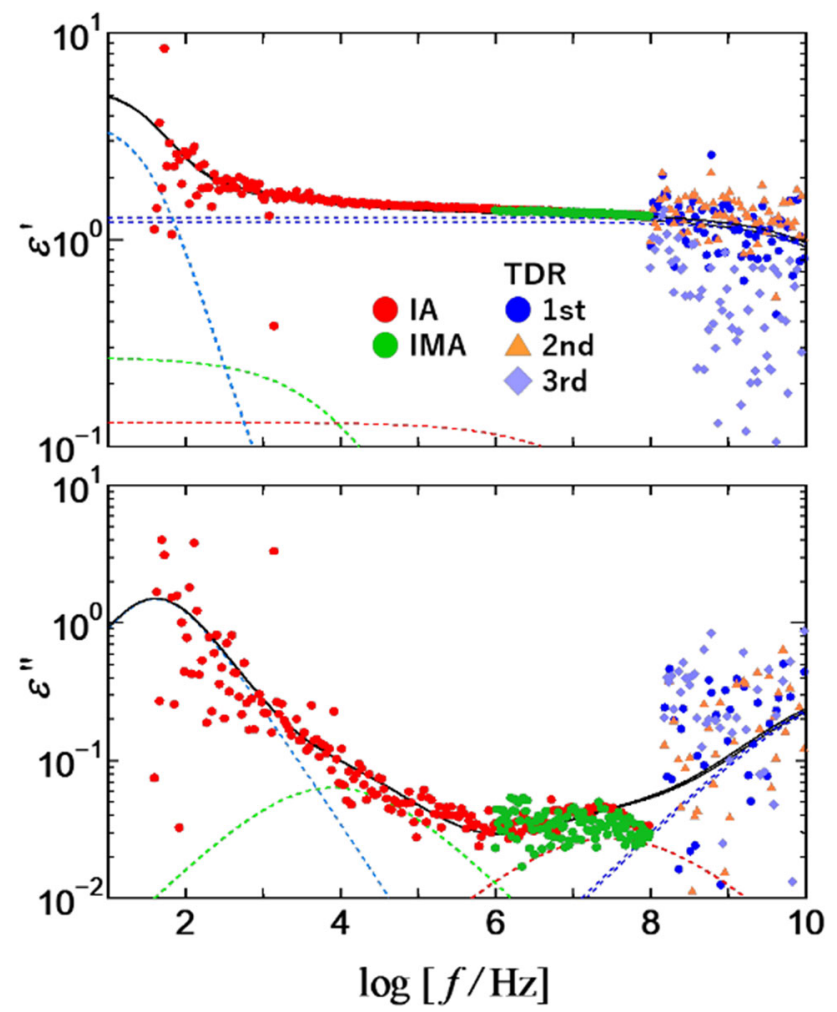

Figure 7 BDS spectrum obtained from three sub-systems in their respective frequency regions for a dried sample of cotton cloth. The solid line was obtained from the fitting procedure of Eq. (4). sample with low water content. Furthermore, the use of logarithmic scale graphs to show the entire data also makes the error remarkable. The validity of the small permittivity values obtained will be further discussed in subsequent paragraphs.

Figure 7 shows that the BDS spectra could be obtained from suitable fitting procedures with a small contribution from the frequency region from $10 \mathrm{MHz}$ up to $1 \mathrm{GHz}$ in the entire BDS spectra. This procedure combined several measuring sub-systems, and it is certainly an advantage of BDS measurements. Figure 7 also clearly shows that the decreasing behavior of the dielectric constant continues in the broad frequency region, from the $\mathrm{kHz}$ to $\mathrm{GHz}$ regions.

Fitting procedures were applied with high-frequency data obtained from the average of three TDR measurements, as shown in Fig. 8. Though the averaging procedures were not sufficiently effective to reduce the experimental error by simple static treatments of the three measurements, the $\mathrm{GHz}, \mathrm{MHz}$, and $\mathrm{kHz}$ processes, with broad distributions of

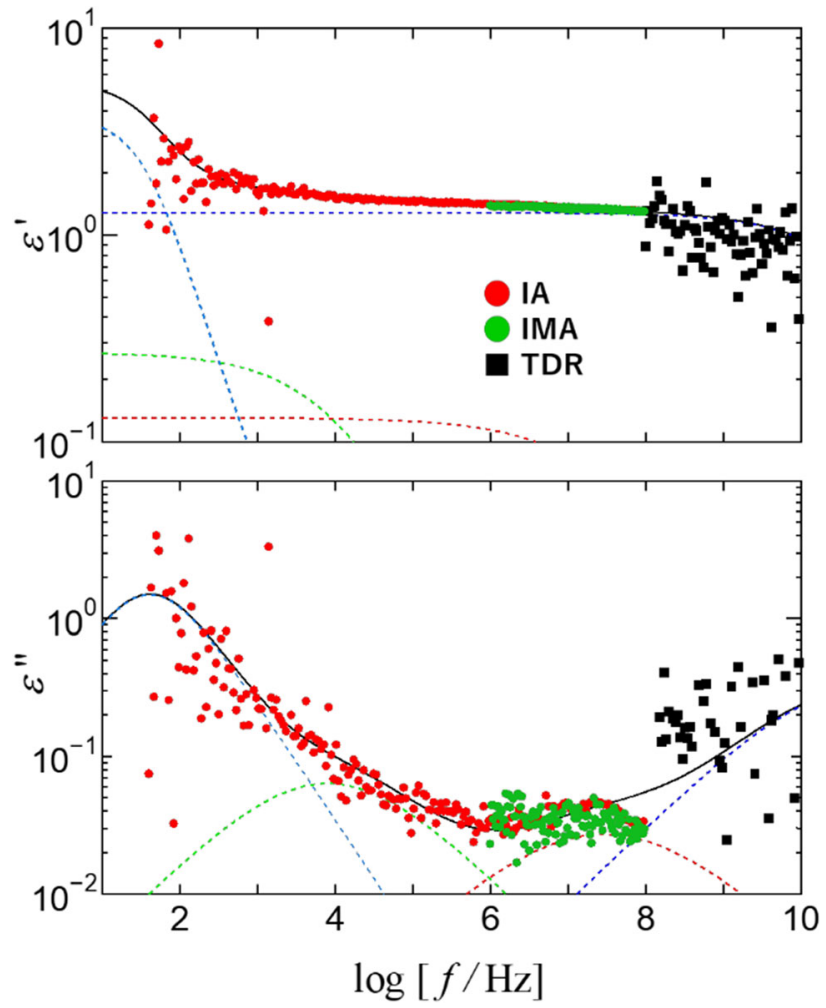

Figure 8 BDS spectrum obtained from three sub-systems in their respective frequency regions for a dry sample of cotton cloth with the average TDR measurement data. The solid line was obtained from the fitting procedure of Eq. (4). 
relaxation time, have overlapped each other in the entire frequency region with the low-frequency process due to electrode polarization.

The relaxation curves obtained by BDS measurements were analyzed by fitting procedures using the following equation:

$$
\begin{aligned}
\varepsilon^{*}= & \varepsilon^{\prime}-j \varepsilon^{\prime \prime} \\
= & \varepsilon_{\infty}+\frac{\Delta \varepsilon_{\mathrm{E}}}{1+\left(j \omega \tau_{\mathrm{E}}\right)^{\beta_{\mathrm{E}}}}+\frac{\Delta \varepsilon_{1}}{1+\left(j \omega \tau_{1}\right)^{\beta_{1}}}+\frac{\Delta \varepsilon_{2}}{1+\left(j \omega \tau_{2}\right)^{\beta_{2}}} \\
& +\frac{\Delta \varepsilon_{3}}{1+\left(j \omega \tau_{3}\right)^{\beta_{3}}}-j \frac{\sigma_{\mathrm{DC}}}{\varepsilon_{0} \omega}, \quad(0<\beta \leq 1)
\end{aligned}
$$

where $\sigma$ is the direct current (dc) conductivity and $\varepsilon_{0}$ is the dielectric constant of vacuum. The subscripts 1 , 2 , and 3 in Eq. (4) indicate the relaxation process around $10 \mathrm{kHz}, 10 \mathrm{MHz}$, and $10 \mathrm{GHz}$, respectively, and the subscript $\mathrm{E}$ indicates the process due to electrode polarization. The relaxation parameters for the $\mathrm{GHz}$ process are listed with those average values in Table 1. In the present paper, we focus on the discussion of water structures from the relaxation parameters obtained for the $\mathrm{GHz}$ process using the fractal concept.

Table 1 indicates a relaxation time value ( 3 ps) that is smaller than that for usual bulk water ( $8 \mathrm{ps})$. It is considered that the small value was easily induced by fitting procedures for the broadening $\mathrm{GHz}$ frequency processes without a clear loss peak in the high-frequency region. Here, we did not use any restrictions to maintain the relaxation time of $3 \mathrm{ps}$ since it was important to confirm a relative change in the dielectric constant and loss for the lower-frequency side of the $\mathrm{GHz}$ process. The $\beta$ value is determined from the slope of the lower-frequency side of the $\mathrm{GHz}$ process in the absorption curve [40].

Considering that the water content of dry cotton cloths kept under typical laboratory condition is about $5 \%$, the values of relaxation strength of the $\mathrm{GHz}$ frequency process of around 1.2-1.3 were still smaller than those expected from a simple proportional relationship with the water content. It is reasonable to consider that these smaller values reflect air included in the cotton cloths and the lowerfrequency shift of dynamic behaviors of water molecules restricted with cellulose chains. In our present model of hydration, we do not need to distinguish the two kinds of dynamic behaviors of water molecules as one from the lower-frequency tail of the $\mathrm{GHz}$ frequency process and another from $\mathrm{MHz}$ processes $[9,41]$. This is because the two broadening relaxation time distributions sufficiently overlap [42]. Though the $\beta$ value of 0.55 is smaller than usual for moist materials, it is difficult to make exact and reasonable comparisons with hydration structures of other materials, as explained for Fig. 1.

Therefore, more detailed discussion of hydration is expected to be obtain from the $\tau-\beta$ diagram in Fig. 9 for the $\mathrm{GHz}$ frequency process of the cotton cloth. Though the $\beta$ value itself means the time correlation behavior of HBN dynamics, the combination with the relaxation time suggests the spatial fractal structures of HBNs as shown by Eq. (2). Abbreviations for the various aqueous materials used in Fig. 9 and related references are shown in the figure caption. A $\beta$ value of 0.55 has been obtained for conventional dispersion systems reported thus far; however, the combination of such small value, 0.55 , and the small relaxation time obtained for $\mathrm{GHz}$ process has not been reported so far. Even if we consider the error, indicated as an arrow, for the relaxation time, the plot for the cotton cloth shown in Fig. 9 still clearly indicates a value of $\beta$ smaller than that of typical materials.

The smaller fractal dimension means broad spatial distribution of $\mathrm{HBN}$ fragmentations with various sizes, which is generally shown as typical behavior of dispersion systems [14]. It is also emphasized that cotton cloth does not show the usual lower-frequency shift of $\mathrm{GHz}$ frequency process that is shown in typical solution systems. The characteristic behavior of cotton cloth is the retaining of HBNs with an aggregation structure of water molecules, even in the dry state, and fragmentations of the HBN at the same
Table 1 Relaxation parameters obtained from three TDR measurements for naturally dried samples with eight sheets of thrice-folded cotton cloth

\begin{tabular}{llll}
\hline Measurement & $\begin{array}{l}\text { Relaxation strength } \\
\Delta \varepsilon\end{array}$ & $\begin{array}{l}\text { Relaxation time } \\
\tau(\mathrm{ps})\end{array}$ & $\begin{array}{l}\text { Relaxation time distribution parameter } \\
\beta\end{array}$ \\
\hline 1st & 1.23 & 3.02 & 0.55 \\
2nd & 1.29 & 3.02 & 0.55 \\
3rd & 1.29 & 3.02 & 0.55 \\
Average & 1.27 & 3.02 & 0.55 \\
\hline
\end{tabular}




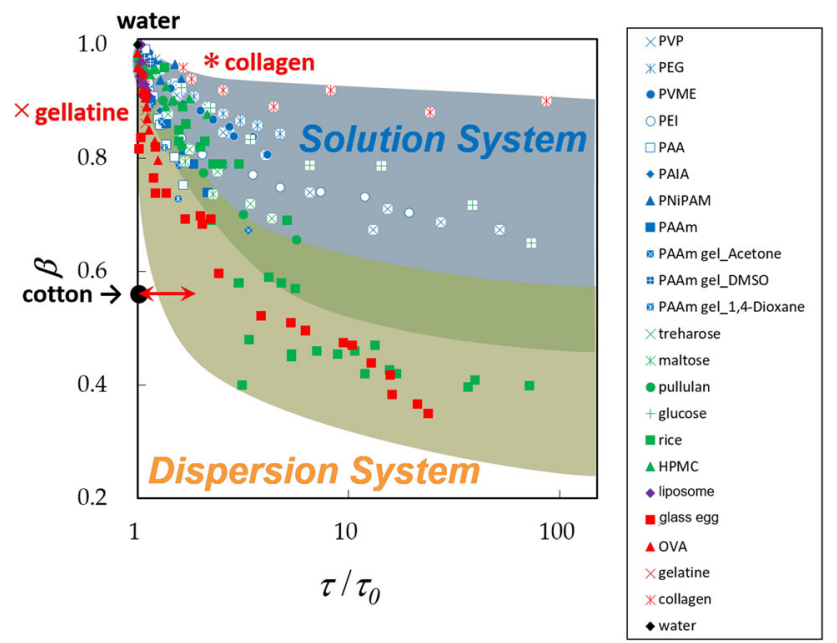

Figure $9 \tau-\beta$ diagram obtained for various aqueous systems and a plot obtained for naturally dried samples of cotton cloth. The various substances used for aqueous mixtures shown in the figure follow commonly used abbreviations as; poly(vinylpyrrolidone) (PVP) [19], poly(ethylene glycol) (PEG) [19], poly(vinyl methyl ether) (PVME) [19], poly(ethylenimine) (PEI) [19], poly(acrylic acid) (PAA) [19], poly(allylamine) (PAlA) [19], Poly(N-isopropylacrylamide) (PNiPAM) [43], poly(acrylamide) (PAAm) [44], PAAm gels in water-acetone mixtures (PAAm gel Acetone) [20, 44], PAAm gels in waterdimethyl sulfoxide mixtures (PAAm gel DMSO) [20], PAAm gels in water-1, 4-dioxane (PAAm gel 1, 4-dioxane) [20], trehalose $[45,46]$, maltose [45, 46], pullulan [47], glucose [45, 46], rice [48], hydroxypropylmethylcellulose (HPMC) [46, 49, 50],1,2dipalmitoylsn-glycero-3-phosphacholine (liposome) [46, 51-53], denatured chicken egg white (glass egg) [46, 54], ovalbumin (OVA) [46, 55-58], gelatin [46, 58], bovine Achilles tendon (collagen) [46, 58, 59]. The red arrow indicates the maximum error estimated from the $\tau$ value.

time. The size of aggregations is probably considered to be more than $1 \mathrm{~nm}$, corresponding to five or six water molecules. The existence of water molecules with different mobilities was also observed by NMR $T_{2}$ relaxation measurements.

Figure 10 shows plots of the intensity, $M(t)$, against the echo time, $t$, obtained from NMR $T_{2}$ measurements with the CPMG method for the humid sample kept under $98 \% \mathrm{RH}$. The echo time dependence of the decay could not be explained by a single exponential function. Thus, the decay curve was expressed by following bi-exponential behavior, given by

$M(t)=A_{f} e^{-\left(\frac{t}{T_{2 f}}\right)}+A_{s} e^{-\left(\frac{t}{T_{2 s}}\right)}$,

where the first and second terms indicate the fast and slow components of the decay, respectively.

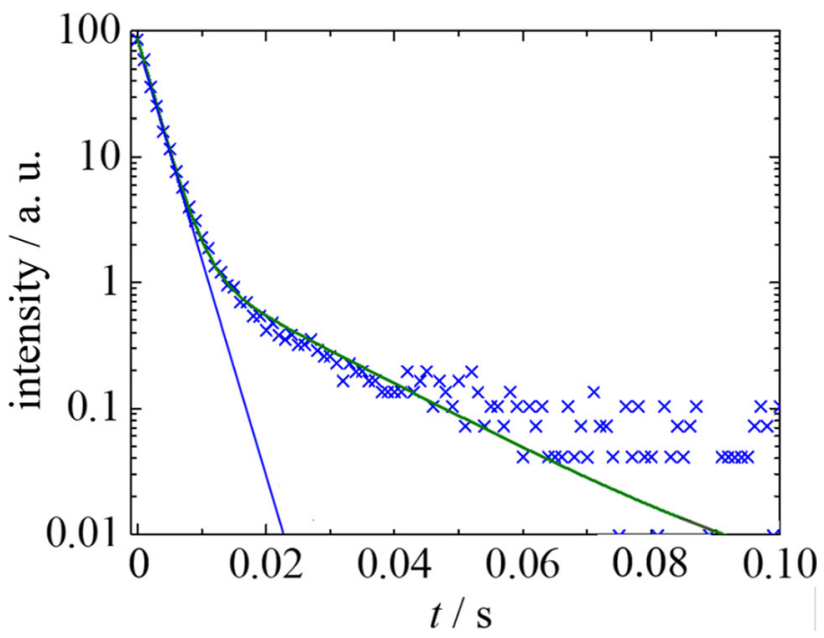

Figure 10 Echo time dependency of the intensity obtained by the CPMG method of $T_{2}$ relaxation time measurements of NMR for the cotton yarn sample kept under 98\% RH. Lines were obtained from the fitting procedure for the fast process with a single exponential function (blue) and the entire decay with a biexponential function (green).

Figure 10 shows that the bi-exponential function describes the decay behavior well, except for the larger echo time region where the signal decays and is hidden in the error.

The bi-exponential analysis applied for all samples with Eq. (5) suggested that $T_{2 \mathrm{~s}}$ and $T_{2 \mathrm{f}}$ values for the humid samples are 16.4 and $2.4 \mathrm{~ms}$, respectively. These values decreased to 9.5 and $0.72 \mathrm{~ms}$ for the dry sample. It is reasonable to consider that the mobility of water molecules interacting with cellulose molecules increases with increase in water content, as shown in typical aqueous systems.

These results also indicate that the dynamic behavior of water molecules cannot be characterized by a simple mean relaxation time. The difference in mobility of these water molecules observed by NMR measurements does not have to quantitatively correspond to the relaxation parameters obtained from BDS measurements. This is because of the nanosecond to picosecond time domain used in dielectric measurements while NMR measurements require a larger time scale, in tens of milliseconds, corresponding to a larger length scale. The NMR measurements should then reflect dynamic behaviors obtained from an average in the larger space and time scales. Nevertheless, the same conclusion of the existence of various mobile properties, or the broad distribution of water molecules, was obtained from 
these two different observation techniques, and their consistent results are significant.

As described above, the present analysis with the $\tau-\beta$ diagram is focused on the plot area rather than on values of the fractal dimension since conventional analysis has often shown large fluctuations of fractal dimension due to mathematical fitting procedures for scattering data. With respect to a database of $\tau-\beta$ diagrams $[14,30]$, the cotton data were more prominent than any dispersion systems previously observed. By contrast, even in the same category of protein-water systems, plots for collagen were obtained in the area for solution systems and plots for gelatin solutions were located in the area for dispersion systems. These results may seem opposing since the more structured collagen shows a more solutionlike result and the coiled state of gelatin indicated a dispersion-like result. However, these results can be interpreted as HBNs in gelatin excluded from the globular protein structure and HBNs remain around collagen molecules and tropocollagen. In the case of collagen, HBNs of water molecules and collagen chains are mutually penetrating [30]. When this model is applied to cotton cloth, it is presumed that HBNs of water molecules cannot penetrate the 1-nmscale microfibrils of cellulose and that $\mathrm{HBNs}$ of water molecules and microfibrils of cellulose cannot penetrate each other, like collagen. This model corresponds well to NMR studies reported by Salmén et al. [8]. The hydration properties of the cotton cloth sample obtained in this study were similar to those for conventional dispersion systems; however, the relaxation time distribution parameters showed an even smaller value. This low fractal dimension indicates heterogeneous hydration in the cotton cloth sample and similar dynamic behaviors for the bulk water, even at a low average moisture content. The cotton cloth sample is more inhomogeneous than usual dispersion systems since various voids filled by air exist, as well as heterogeneous cellulosic fibers. There exist local high water content parts in the sample, even for the low average water content sample, and the HBN fragments exhibiting bulk-like water contribute to the $\mathrm{GHz}$ frequency process. By contrast, HBN fragments smaller than the correlation length of HBN in bulk water have a low hydrogen bond density, contribute to the lower-frequency component of the relaxation time distribution, and exhibit an overlap with water molecules bound to cellulose in the same relaxation frequency range.
Even when the cotton cloth is naturally dried from the large water content, water molecules are heterogeneously removed. The remaining water molecules aggregate to retain the HBN fragment, which contributes to the $\mathrm{GHz}$ process, and some water molecules bound to cellulose form structures. However, the $\mathrm{GHz}$ process has not been treated in conventional investigations for low water content substances, and the water structure related to the $\mathrm{GHz}$ process extending to such a low-frequency region was evaluated for the first time. Considering consistency with the hydration model proposed by Igarashi et al. [1-3], the drying process of cotton cloth broadens the $\mathrm{GHz}$ process, and the size distribution of HBN fragments also shows broadening. The smaller size of the HBN fragments contributes to the lower-frequency components of the $\mathrm{GHz}$ process. Water molecules interacting with the cellulose chains via $\mathrm{HB}$ observed at $\sim 1 \mathrm{MHz}$ and those included in the low-frequency tail of the $\mathrm{GHz}$ process cannot be distinguished because of the overlap of the two relaxation processes. However, the model of the stiffening mechanism of cotton fabrics suggests that these restricted water molecules are closely related to the stiffening mechanism of the cotton fabric. When water molecules are removed by mechanical stimulus or further drying from the stiff fabrics in the dry state, the cotton fabric is rejuvenated and the stiffening effect is loosened. The slow component of water molecules takes a single HB state with the cellulose molecules. More detailed interpretations are expected to be obtained from further investigations with the same BDS analytical techniques and other complementary observation techniques for water structures of cotton materials modified by treatments with additives, for example.

The fractal analysis shown in the present work suggests dynamic behaviors and locations of water molecules in the materials. In various areas wherein water structures of aqueous systems, such as moist materials, biomaterials, and cement materials, are treated, the present analytical technique of BDS using fractal analysis with the $\tau-\beta$ diagram is expected to be an effective and universal tool to clarify details of hydration mechanisms. 


\section{Conclusions}

In the present work, the water structure of naturally dried cotton cloth was investigated by BDS and fractal analysis of spatial distribution of HBNs with various fragment sizes performed with a $\tau-\beta$ diagram. The analysis of the $\mathrm{GHz}$ process revealed that the mobility of water molecules significantly fluctuated, reflecting variety in the agglutination/dispersion state of water molecules in the cotton cloth. Comparisons of the $\beta$ value (0.55) with the smaller relaxation time with a database obtained from our previous studies suggest that water molecules involved in HBN fragments with various sizes tend to disperse widely in a sample while aggregating much stronger than other substances or conditions currently reported. It is expected that the present characterization technique providing more detailed insights into the widely distributed water structures that have not been covered in previous studies can be applied to the evaluation of various properties of cotton cloth samples in future developments of material processing and fabric softener. It was also shown that our analytical technique is effective for universal analysis of water structures for moist materials, even for those in which there exist serious difficulties in measurements of the absolute value of permittivity.

\section{Acknowledgements}

The authors are grateful to Mr. Taku Mimura, Vice President of Global R\&D for Material Science Research at Kao Corporation, Japan, for financial support.

\section{Declarations}

Conflict of interest The authors declare no conflict of interest.

Open Access This article is licensed under a Creative Commons Attribution 4.0 International License, which permits use, sharing, adaptation, distribution and reproduction in any medium or format, as long as you give appropriate credit to the original author(s) and the source, provide a link to the Creative Commons licence, and indicate if changes were made. The images or other third party material in this article are included in the article's Creative Commons licence, unless indicated otherwise in a credit line to the material. If material is not included in the article's Creative Commons licence and your intended use is not permitted by statutory regulation or exceeds the permitted use, you will need to obtain permission directly from the copyright holder. To view a copy of this licence, visit http://creativecommons.org/licen ses $/$ by $/ 4.0 /$.

\section{References}

[1] Igarashi T, Morita N, Okamoto Y, Nakamura K (2016) Elucidation of softening mechanism in rinse cycle fabric softeners. Part 1: Effect of hydrogen bonding. J Surfactants Deterg 19:183-192. https://doi.org/10.1007/s11743-015-17 $32-4$

[2] Igarashi T, Nakamura K, Hoshi M, Hara T, Kojima H, Ito M, Ikeda R, Okamoto Y (2016) Elucidation of softening mechanism in rinse-cycle fabric softeners. Part 2: uneven adsorption-the key phenomenon to the effect of fabric softeners. J Surfactants Deterg 19:759-773. https://doi.org/10. 1007/s11743-016-1815-x

[3] Igarashi T, Hoshi M, Nakamura K, Kaharu T, Murata K (2019) Direct observation of bound water on cotton surfaces by atomic force microscopy and atomic force microscopy-infrared spectroscopy. J Phys Chem C 124:4196-4201. https://doi.org/10.1021/acs.jpcc.0c00423

[4] Petridis L, O’Neill HM, Johnsen M, Fan B, Schulz R, Mamontov E, Maranas J, Langan P, Smith JC (2014) Hydration control of the mechanical and dynamical properties of cellulose. Biomacromol 15:4152-4159. https://doi.or $\mathrm{g} / 10.1021 / \mathrm{bm} 5011849$

[5] Kulasinski K, Keten S, Churakov SV, Guyer R, Carmeliet J, Derome D (2014) Molecular mechanism of moisture-induced transition in amorphous cellulose. ACS Macro Lett 3:1037-1040. https://doi.org/10.1021/mz500528m

[6] Paajanen A, Ceccherini S, Maloney T, Ketoja JA (2019) Chirality and bound water in the hierarchical cellulose structure. Cellulose 26:5877-5892. https://doi.org/10.1007/ s10570-019-02525-7

[7] Lindh EL, Terenzi C, Salmén L, Furó I (2017) Water in cellulose: evidence and identification of immobile and mobile adsorbed phases by $2 \mathrm{H}$ MAS NMR. Phys Chem Chem Phys 19:4360-4369. https://doi.org/10.1039/ C6CP08219J

[8] Salmén L, Stevanic JS (2018) Effect of drying conditions on cellulose microfibril aggregation and "hornification." Cellulose 25:6333-6344. https://doi.org/10.1007/s10570-018-203 9-1 
[9] Zhao H, Chenb Z, Dua X, Chena L (2019) Contribution of different state of adsorbed water to the sub-Tg dynamics of cellulose. Carbohyd Polym 210:322-331. https://doi.org/10. 1016/j.carbpol.2019.01.087

[10] Furusawa K, Dobashi T, Morishita S, Oyama M, Hashimoto T, Shinyashiki N, Yagihara S, Nagasawa N (2005) Structural and kinetic modification of aqueous hydroxypropylmethylcellulose (HPMC) induced by electron beam irradiation. Phys A 353:9-20. https://doi.org/10.1016/j.physa.2004.12. 068

[11] Sudo S (2011) Dielectric Properties of the free water in hydroxypropyl cellulose. J Phys Chem B 115:2-6. https://d oi.org/10.1021/jp104950q

[12] Kremer F, Schoenhals A (eds) (2012) Broadband dielectric spectroscopy. Springer-Verlag, Berlin

[13] Raicu V, Feldman Y (eds) (2015) Dielectric relaxation in biological systems. Oxford University Press, Oxford

[14] Yagihara S (2015) Dynamics of water, biomaterials, and skin investigated by means of dielectric relaxation spectroscopy. In: Dobashi T, Kita R (eds) Nano/micro science and technology in biorheology: principles, methods, and applications. Springer, Tokyo, pp 183-213

[15] Kaatze U (2018) Water, the special liquid. J Mol Liq 259:304-318. https://doi.org/10.1016/j.molliq.2018.03.038

[16] Fukasawa T, Sato T, Watanabe J, Hama Y, Kunz W, Buchner R (2005) Relation between dielectric and low-frequency Raman spectra of hydrogen-bond liquids. Phys Rev Lett 95:1-4. https://doi.org/10.1103/PhysRevLett.95.197802

[17] Buchner R, Barthel J, Stauber J (1999) The dielectric relaxation of water between $0{ }^{\circ} \mathrm{C}$ and $35^{\circ} \mathrm{C}$. Chem Phys Lett 306:57-63. https://doi.org/10.1016/S0009-2614(99)00455-8

[18] Kaatze U (1975) Dielectric relaxation in aqueous solutions of polyvinylpyrrolidone. Adv Mol Relax Processes 7:71-85. https://doi.org/10.1016/0001-8716(75)80015-0

[19] Shinyashiki N, Arita I, Yagihara S, Mashimo S (1998) Dynamics of water in a polymer matrix studied by a microwave dielectric measurement. J Phys Chem B 102:3249-3251. https://doi.org/10.1021/jp9729627

[20] Saito H, Kato S, Matsumoto K, Umino Y, Kita R, Shinyashiki N, Saito H, Kato S, Matsumoto K, Umino Y, Kita R, Shinyashiki N, Yagihara S, Fukuzaki M, Tokita M (2018) Dynamic behaviors of solvent molecules restricted in poly (acryl amide) gels analyzed by dielectric and diffusion NMR spectroscopy. Gels 4:56. https://doi.org/10.3390/ge $1 s 4030056$

[21] Shinyashiki N, Imoto D, Yagihara S (2007) Broadband dielectric study of dynamics of polymer and solvent in poly (vinyl pyrrolidone)/normal alcohol mixtures. J Phys Chem B 111:2181-2187. https://doi.org/10.1021/jp065414e
[22] Hirata T, Matsuno H, Kawaguchi D, Inutsuka M, Hirai T, Tanaka M, Tanaka K (2017) Dynamics of a bioinert polymer in hydrated states by dielectric relaxation spectroscopy. Phys Chem Chem Phys 19:1389-1394. https://doi.org/10.1039/ C6CP07322K

[23] Ryabov YE, Feldman Y (2002) Novel approach to the analysis of the non-Debye dielectric spectrum broadening. Phys A Stat Mech Appl 314(1-4):370-378. https://doi.org/ 10.1016/S0378-4371(02)01076-2

[24] Ryabov YE, Feldman Y, Shinyashiki N, Yagihara S (2002) The symmetric broadening of the water relaxation peak in polymer-water mixtures and its relationship to the hydrophilic and hydrophobic properties of polymers. J Chem Phys 116:8610-8615. https://doi.org/10.1063/1.1471551

[25] Puzenko A, Ishai PB, Feldman Y (2010) Cole-cole broadening in dielectric relaxation and strange kinetics. Phys Rev Lett 105:03760114. https://doi.org/10.1103/PhysRevLett.10 5.037601

[26] Levy E, Puzenko A, Kaatze U, Ishai PB, Feldman Y (2012) Dielectric spectra broadening as the signature of dipolematrix interaction. I. Water in nonionic solutions. J Chem Phys 136:114502. https://doi.org/10.1063/1.3687914

[27] Levy E, Puzenko A, Kaatze U, Ishai PB, Feldman Y (2012) Dielectric spectra broadening as the signature of dipolematrix interaction. II. Water in ionic solutions. J Chem Phys 136:114503. https://doi.org/10.1063/1.3691183

[28] Kaatze U, Behrends R, Pottel R (2002) Hydrogen network fluctuations and dielectric spectrometry of liquids. J NonCryst Solids 305:19-28. https://doi.org/10.1016/S0022-309 3(02)01084-0

[29] Maruyama Y, Numamoto Y, Saito H, Kita R, Shinyashiki N, Yagihara S, Fukuzaki M (2014) Complementary analyses of fractal and dynamic water structures in protein-water mixtures and cheeses. Colloids Surf A Physicochem Eng Asp 440:42-48. https://doi.org/10.1016/j.colsurfa.2012.10.051

[30] Yagihara S, Kita R, Shinyashiki N, Saito H, Maruyama Y, Kawaguchi T, Shoji K, Saito T, Aoyama T, Shimazaki K, Matsumoto K, Fukuzaki M, Masuda H, Hiraiwa S, Asami K, Tokita M (2019) Physical meanings of fractal behaviors of water in aqueous and biological systems with open-ended coaxial electrodes. Sensors 19:2606. https://doi.org/10.3390/ s19112606

[31] Kawaguchi T, Kita R, Shinyashiki N, Yagihara S, Fukuzaki M (2018) Physical properties of tofu gel probed by water translational/rotational dynamics. Food Hydrocolloids 77:474-481. https://doi.org/10.1016/j.foodhyd.2017.10.025

[32] Cole KS, Cole RH (1941) Dispersion and absorption in dielectrics I. Alternating current characteristics. J Chem Phys 9:341. https://doi.org/10.1021/acs.jpcc.0c00423 
[33] Debye P (1913) Zur Theorie der anomalen dispersion im Gebiete der langwelligen elektrischen Strahlung. Verh Dtsch Phys Ges 15:777-793

[34] Williams G, Watts DC (1971) Non-symmetrical dielectric relaxation behaviour arising from a simple empirical decay function. Trans Faraday Soc 66:80-85. https://doi.org/10.1 039/TF9706600080

[35] Havriliak S, Negami A (1966) Complex plane analysis of $\alpha-$ dispersions in some polymer systems. J Polym Sci C 14:99-117. https://doi.org/10.1002/polc.5070140111

[36] Abe F, Nishi A, Saito H, Asano M, Watanabe S, Kita R, Shinyashiki N, Yagihara S, Fukuzaki M, Sudo S, Suzuki Y (2017) Dielectric study on hierarchical water structures restricted in cement and wood materials. Meas Sci Technol 28:044008. https://doi.org/10.1088/1361-6501/aa5c65

[37] Shoji K, Saito T, Kita R, Shinyashiki N, Yagihara S, Fukuzaki M, Ohzono T, Nishimura S, Hayashi M, Tanaka H (2018) Dynamics and aggregate structuring of water molecules in edible oil analyzed by dielectric spectroscopy. Trans Mat Res Soc Japan 43(3):201-204. https://doi.org/10.14723/ tmrsj.43.201

[38] Maruyama Y, Kamata H, Watanabe S, Kita R, Shinyashiki N, Yagihara S (2019) Electric-field penetration depth and dielectric spectroscopy observations of human skin. Skin Res Technol 26:255-262. https://doi.org/10.1111/srt.12788

[39] Farrar TC, Becker ED (1971) Pulse and fourier transform NMR: introduction to theory and methods, English. Academic Press, New York

[40] Sudo S, Suzuki Y, Abe F, Hori Y, Nishi T, Kawaguchi T, Saito H, Yagihara S (2018) Investigation of the molecular dynamics of restricted water in wood by broadband dielectric measurements. J Mater Sci 53(6):4645-4654. https://doi.org/ 10.1007/s10853-017-1824-9

[41] Yagihara S, Nozaki R, Mashimo TS (1983) Evaluation of dielectric permittivity by de transient current. J Chem Phys 79(5):2419-2422. https://doi.org/10.1063/1.446049

[42] Kaatze U (2011) Bound water: Evidence from and implications for the dielectric properties of aqueous solutions. J Mol Liq 162:105-112. https://doi.org/10.1016/j.molliq.2011.06. 012

[43] Nakano S, Sato Y, Kita R, Shinyashiki N, Yagihara S, Sudo S, Yoneyama M (2012) Molecular dynamics of Poly(Nisopropylacrylamide) in protic and aprotic solvents studied by dielectric relaxation spectroscopy. J Phys Chem B 116:775-781. https://doi.org/10.1021/jp210376u

[44] Yamada G, Hashimoto T, Morita T, Shinyashiki N, Yagihara S, Tokita M (2001) Dielectric study on dynamics for volume phase transition of PAAm gel in Acetone-Water system. Trans Mater Res Soc Japan 26:701-704
[45] Mashimo S, Miura N, Umehara T (1992) The structure of water determined by microwave dielectric study on water mixtures with glucose, polysaccharides, and L-ascorbic acid. J Chem Phys 97:6759-6767. https://doi.org/10.1063/1. 46365

[46] Matsumoto K (2009) Fractal structure analysis of aqueous mixtures by dielectric spectroscopy (Japanese). MSc Dissertation, Graduate School of Science, Tokai University.

[47] Kishikawa Y, Seki Y, Shingai K, Kita R, Shinyashiki N, Yagihara S (2013) Dielectric relaxation for studying molecular dynamics of Pullulan in water. J Phys Chem B 117:9034-9041. https://doi.org/10.1021/jp403606r

[48] Yagihara S, Oyama M, Inoue A, Asano M, Sudo S, Shinyashiki N (2007) Dielectric relaxation measurement and analysis of restricted water structure in rice kernels. Meas Sci Technol 18:983-990. https://doi.org/10.1088/0957-0233/18/ $4 / 004$

[49] Kita R, Kaku T, Ohashi H, Kurosu T, Iida M, Yagihara S, Dobashi T (2003) Thermally induced coupling of phase separation and gelation in an aqueous solution of hydroxypropylmethylcellulose (HPMC). Phys A 319:56-64. http s://doi.org/10.1016/S0378-4371(02)01523-6

[50] Hashimoto T, Yamamura M, Shigematsu T, Shioya S, Oyama M, Shimomura M, Miura N, Shinyashiki N, Yagihara S, Hayashi Y, Tokita M, Nishida H, Terao K, Dobashi T, Yoshii F (2003) Dielectric study on polymer gel in frozen state. Trans Mater Res Soc Japan 28:997-1000

[51] Miyairi H, Shinyashiki N, Yagihara S (1997) Dielectric relaxation of dipalmitoyl-phosphatydylcholine liposome in aqueous solution. Rept Progr Polym Phys Japan 40:621-624

[52] Hosoi Y, Kita R, Shinyashiki N, Yagihara S (2010) Dielectric study of liposome dispersion using statistical analysis of Jitter. Trans Mater Res Soc Japan 35:861-864

[53] Kundu SK, Choe S, Sasaki K, Kita R, Shinyashiki N, Yagihara S (2015) Relaxation dynamics of liposome in an aqueous solution. Phys Chem Chem Phys 17:18449-18455. https://doi.org/10.1039/C5CP01334H

[54] Hayashi Y, Miura N, Shinyashiki N, Yagihara S, Mashimo S (2000) Globule-coil transition of denatured globular protein investigated by a microwave dielectric technique. Biopolymers 54:388-397. https://doi.org/10.1002/1097-0282(20001 1) $54: 6 \% 3 \mathrm{c} 388:: A I D-B I P 30 \% 3 \mathrm{e} 3.0 . \mathrm{CO} ; 2-\mathrm{K}$

[55] Miura N, Asaka N, Shinyashiki N, Mashimo S (1994) Microwave dielectric study on bound water of globule proteins in aqueous solution. Biopolymers 34:357-364. https://d oi.org/10.1002/bip.360340307

[56] Miura N, Hayashi Y, Shinyashiki N, Mashimo S (1995) Observation of unfreezable water in aqueous solution of globule protein by microwave dielectric measurement. 
Biopolymers 36:9-16. https://doi.org/10.1002/bip. $360360103 \mathrm{~g}$

[57] Matsumoto K, Shimazaki K, Kitamura K, Kita R, Shinyashiki N, Yagihara S (2008) Geometrical feature of fractal structure expression and characterization of aqueous mixtures by dielectric spectroscopy (Japanese). Proc School Sci Tokai Univ 43:127-143

[58] Hayashi Y, Shinyashiki N, Yagihara S (2002) Dynamical structure of water around biopolymers investigated by microwave dielectric measurements via time domain reflectometry. J Non-Crist Solids 305:328-332. https://doi. org/10.1016/S0022-3093(02)01113-4

[59] Shinyashiki N, Asaka N, Mashimo S, Yagihara S, Sasaki N (1990) Microwave dielectric study on hydration of moist collagen. Biopolymers 29:1185-1191. https://doi.org/10.10 02/bip.360290809

Publisher's Note Springer Nature remains neutral with regard to jurisdictional claims in published maps and institutional affiliations. 